\title{
Acute stroke management pathway during Coronavirus-19 pandemic
}

\author{
Claudio Baracchini ${ }^{1}$ - Alessio Pieroni ${ }^{1}$ (1) $\cdot$ Federica Viaro ${ }^{1} \cdot$ Vito Cianci $^{2} \cdot$ Anna M. Cattelan $^{3} \cdot$ Ivo Tiberio $^{4}$. \\ Marina Munari ${ }^{5} \cdot$ Francesco Causin $^{6}$
}

Received: 19 March 2020 / Accepted: 25 March 2020 / Published online: 9 April 2020

(C) Fondazione Società Italiana di Neurologia 2020

\begin{abstract}
Since the outbreak of the COVID-19 epidemic which in our region, Veneto (Italy), dates back to February, we were confronted with several challenges, but with a constant aim of keeping our Stroke Unit COVID-free. For this reason, in addition to creating a dedicated hot-spot as a pre-triage just outside the Emergency Department, together with the Neuroradiology Unit we obtained a mobile CT unit that could be used by COVID-positive or COVID-suspected patients. Furthermore, thanks to the collaboration with colleagues from different specialties (Infectious Disease, Internal Medicine, Intensive Care, Emergency Medicine), dedicated areas for COVID patients were activated. This led to a substantial change of our acute stoke management pathway. As the number of COVID patients increased, and the WHO declared a state of pandemic, this new stroke pathway has been fully tested. We would like to share our experience and send a clear message to keep a high attention on stroke as an emergency condition, because we have observed a decreased number of patients with minor strokes and TIAs, longer onset-to-door and door-totreatment times for major strokes, and a reduced number of transfers from spokes. We strongly believe that the general population and family doctors are rightly focused on COVID. However, to remain at home with stroke symptoms does not mean to "stay safe at home".
\end{abstract}

Keywords Acute stroke $\cdot$ Stroke Unit · Coronavirus · COVID-19

The CORONAVIRUS-19 pandemic is changing our personal and professional lives. While this is occurring, people continue to have "traditional" diseases and in certain instances need a rapid access to the Emergency Department (ED). Since the end of February, we have witnessed an overflow of patients, with the real risk of spreading the viral infection. For this reason, at our University Hospital with a catchment area of about 950,000 people, we have decided to elaborate a more articulate pathway for acute stroke patients, before admitting them to our Stroke Unit.

Alessio Pieroni

pieroni.md@gmail.com

1 Stroke Unit and Neurosonology Laboratory, Padua University Hospital, Padua, Italy

2 Emergency Department, Padua University Hospital, Padua, Italy

3 Tropical and Infectious Diseases Unit, Padua University Hospital, Padua, Italy

4 Intensive Care Unit, Padua University Hospital, Padua, Italy

5 Neuro-Intensive Care Unit, Padua University Hospital, Padua, Italy

6 Neuroradiology Unit, Padua University Hospital, Padua, Italy
On arrival to the ED, eligibility for hyper-acute therapies is usually evaluated by the stroke team who facilitates rapid transit to the Stroke Unit and/or Neuroradiology Unit where treatment can be effectively delivered. This pathway also accommodates a standardized approach for inter-hospital transfer, management of transient ischemic attack, and an acute stroke care protocol applicable to all patients beyond the hyper-acute phase.

Since the outbreak of the COVID epidemic, a triage protocol has been activated in a hotspot of the ED in order to assess each stroke patient for suspected or confirmed COVID-19 infection. Here, patients and healthcare personnel must wear recommended personal protection equipment (PPE) such as respirators, eye protection, gowns, and gloves [1-3].

When the patient is already known to be COVID-19 positive, he undergoes cerebral CT, CT angiography (CTA), and $\mathrm{CT}$ perfusion (CTP) on a high-performance mobile $\mathrm{CT}$ unit positioned just outside the ED. Patients deemed eligible for intravenous thrombolysis follow the standard protocol. If the ischemic stroke is not caused by a large vessel occlusion, the patient is transferred to a special unit of the Infectious Disease ward or to a dedicated medical ward where he is clinically monitored by the stroke team and by the internist. Conversely, if the ischemic stroke is determined by a large 


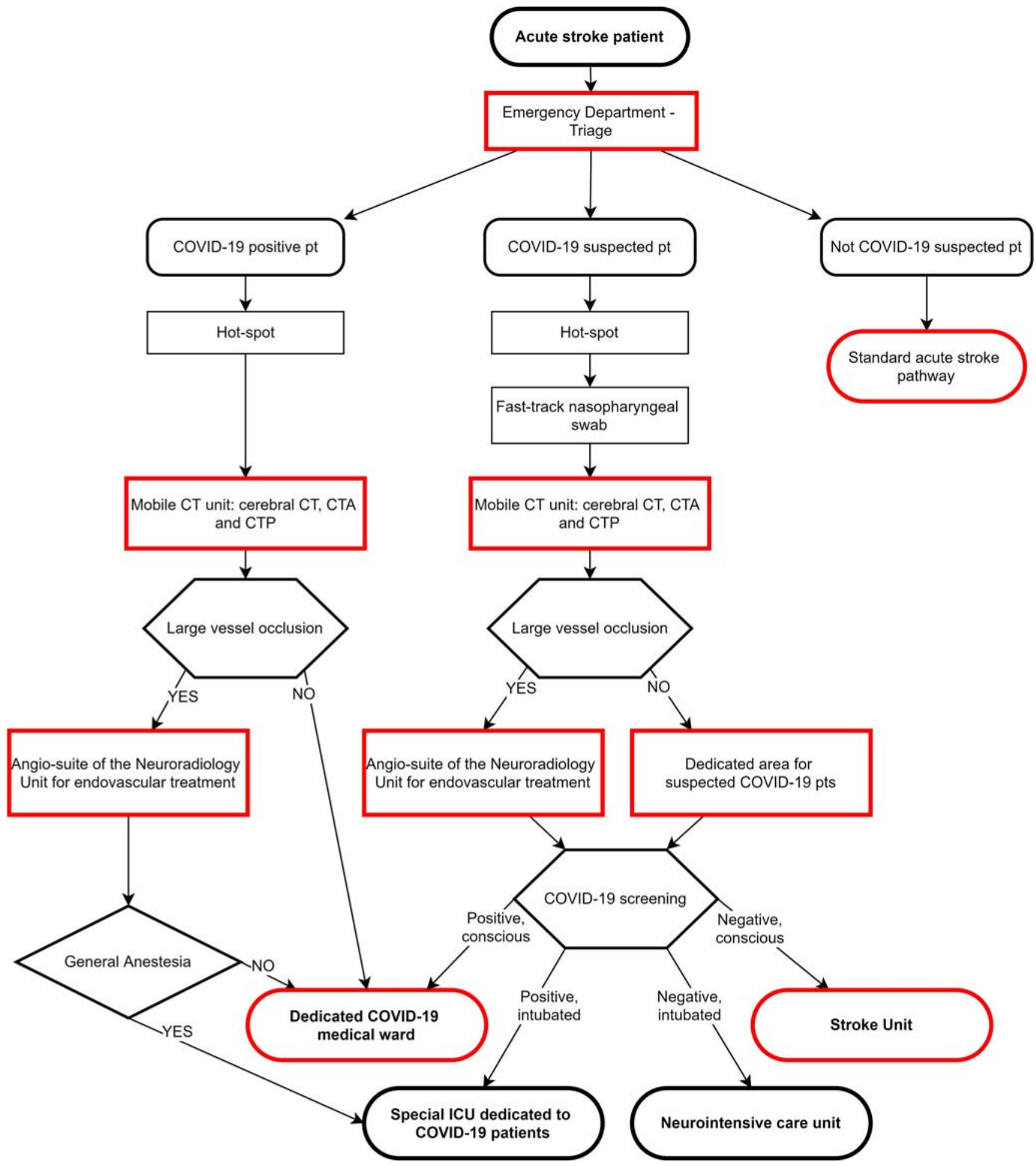

Fig. 1 Acute stroke pathway during Coronavirus-19 pandemic; red boxes indicate stroke team care

vessel occlusion, the patient is transferred to the Angio suite of the Neuroradiology Unit for endovascular treatment, and the personnel is pre-alerted for adopting adequate PPEs. At the end of the procedure, according to the type of anesthesia (i.e., conscious sedation or general anesthesia), the patient is transferred to the Infectious Disease ward or to a special Intensive
Care Unit (ICU) dedicated to COVID-19 patients. In all cases, the stroke team is responsible for the stroke care of the patient.

When the patient is suspected to have COVID-19 infection because of current fever or recent fever and/or flu-like syndrome, nasopharyngeal swabs are taken from the nasopharynx and from each nostril. Noteworthy, the results of this test are 
not rapidly available but take at least one hour. Meanwhile, the patient undergoes cerebral CT, CTA, and CTP on a mobile CT unit positioned just outside the ED. Patients deemed eligible for intravenous thrombolysis follow the standard protocol. If there is no indication for thrombectomy, the patient waits for the result of the COVID-19 screening in a dedicated area, where he is clinically monitored by the stroke team. Only if the COVID-19 screening is negative, the patient is transferred to the Stroke Unit. However, if the COVID-19 screening is positive, the patient is transferred to a dedicated COVID-19 medical ward. When the ischemic stroke is caused by a large vessel occlusion, the patient is transferred to the Angio suite of the Neuroradiology Unit for endovascular treatment, and the personnel is pre-alerted for adopting adequate PPEs. If the COVID-19 screen is negative, the patient is transferred to the Stroke Unit or to the Neuro-ICU according to the type of anesthesia, as reported above. If COVID-19 screen is positive and the procedure has been performed under conscious sedation, the patient is transferred to a special unit of the Infectious Disease ward where he is clinically monitored by the stroke team and by the internist. On the other hand, if thrombectomy is performed under general anesthesia, the patient is transferred to a special ICU dedicated to COVID-19 patients. In all cases, the stroke team is responsible for the stroke care of the patient (Fig. 1).

Currently, the general population, family doctors, and emergency services are rightly focused on COVID-19, and regard the hospital as a non-secure place for elderly, hypertensive, diabetic, obese, and cardiopathic patients because they have a higher mortality rate in case of COVID-19 infection. Alas, these are patients also at higher stroke risk. Compared with the same period in 2019, we have observed a half of minor strokes, TIAs, and transfers from spokes, along with longer onset-to-door and door-to-treatment times for major strokes. As a result, the number of patients who have undergone intravenous thrombolysis or bridging therapy (combined intravenous and thrombectomy) is decreased ($26 \%$ and $-30 \%$ respectively), while the number of primary thrombectomies is increased by $41 \%$ : most of these patients had very serious strokes arriving late, sometimes too late.

We believe that this protocol, based on currently available information about COVID-19, can be adopted by all Comprehensive Stroke Unit hospitals and accessible on a 24/7 basis. There are several advantages with this pathway, namely a protection of our Stroke Unit and personnel from COVID-19 infection, a continuous open access to all possible stroke victims including those coming from spoke hospitals. Finally, in these times of COVID-19 turbulence, this protocol might serve as a safe guide for all caregivers in order not to forget that stroke is a medical emergency and to keep paying a high attention even when symptoms are minor.

\section{Compliance with ethical standards}

Conflict of interest The authors declare that they have no conflict of interest.

Ethical approval None.

\section{References}

1. World Health Organization www.who.int/emergencies/diseases/ novel-coronavirus-2019

2. European Center for Disease Prevention and Control www.ecdc. europa.eu/en

3. Center for Disease Control and Prevention (CDC) www.cdc.gov/ coronavirus/2019-ncov

Publisher's note Springer Nature remains neutral with regard to jurisdictional claims in published maps and institutional affiliations. 